\title{
Avaliação do selamento apical em dentes obturados pela técnica da condensação lateral híbrida, de Tagger e T hermafil
}

\author{
Erica Carvalho ${ }^{1}$ \\ Jorge Andrade únior ${ }^{1}$

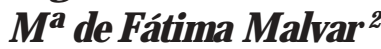 \\ Sílvio Albergaria ${ }^{3}$
}

\begin{abstract}
Resumo
O selamento do sistema decanais radicularesénecessário para a manutenção da desinfecção obtida no preparo químico mecânico, favorecendo o processo de reparo dostecidos periapicais. D iversas técnicas de obturação ecimentostêm sido propostas para atender a esse requisito. Estetrabal ho se propõea avaliar o sel amento apical em dentes obturados pela técnica da condensação lateral, híbrida deTagger eT hermafill, mediantea observação da infiltração do corante azul de metileno. Foram utilizados 40 incisivos centrai ssuperiores, divididos em 3 grupos experimentais, conformeatécnica de obturação empregada, e2 grupos-controle. 0 sresultados obtidosindicam não haver diferença estatisticamente significante quanto à infiltração apical do coranteentreas trêstécnicas de obturação.
\end{abstract}

Palavras-chave: Endodontia - métodos, obturação - canais radiculares, infiltração dentária

INTRODUÇÃO E REVISÃO DE LITERATURA

A obturação do sistema de canais radiculares visa a eliminar os espaços vazios anteriormente ocupados pela polpa, proporcionando maior facilidade no processo de reparação tecidual. 0 selamento endodôntico promove o controle dos microorganismos, impedindo a sua colonização e a invasão em tecidos vizinhos, além de controlar seu potencial de virulência. A presença de fluido tecidual e de microorganismos em contato com o cimento endodôntico podem solubilizá-lo, permitindo uma infiltração que desencadeará um processo inflamatório.
Independentemente da técnica de obturação empregada, a obtenção de um selamento hermético no terço apical é mais difícil em relação aos terços médio e cervical (LEO N ARD O ; LEAL, 1998; VALE; NEUVALD; BRAM AN TE, 2001; ESTRELA, 2004).

A condensação lateral, idealizada por Callahans, em 1914, é a técnica de obturação mais utilizada e conhecida, principalmente de vido à simplicidade de sua execução e aos excelentes resultados clínicos. Entretanto, existem inconvenientes dessa técnica, como a impossibilidade de obtenção de uma obturação tridimensional, a falta de homogeneidade, 0 grande consumo de material, o tempo despen-

\footnotetext{
1 Especialista em Endodontia

2 Prof. a Adjunta, M estre em Clínica O dontológica - Faculdade de O dontologia - UFBA. Salvador - BA

3 Prof. Titular, Livre D ocente em Endodontia-Faculdade de O dontologia - UFBA. Salvador - BA
}

Correspondência para / Correspondence to:

Sílvio Albergaria

Rua João Bião de Cerqueira, 175, apt. 1003 - Pituba

41.830-580 Salvador-Bahia-Brasil

E-mail: silvioalbergaria@gmail.com 
dido, o selamento apical deficiente e a má adaptação do material obturador às paredes dentinárias (FREITAS et al., 1996; M IRAN ZI et al., 2000; JARRETT et al., 2004).

Siqueira Jr, Garcia Filho e Fraga (1993) alertam que a intensificação da força de condensação não proporciona um melhor selamento apical, podendo ser executado um espaçamento sem pressões abusivas, para evitar a ocorrência de fraturas verticais radiculares.

Em 1978, Johnson descreve uma técnica de obturação que emprega um carreador metálico envolvido por uma guta percha que, ao ser aquecida, plastificava-se, sendo transportada para 0 interior do canal radicular. Posteriormente, seguindo essa metodologia, foi introduzida a técnica de obturação termoplastificada, comercializada com o nome de Sistema Thermafil (Tulsa D ental Products, Tulsa, 0 kla.). Tal sistema é constituído por um carregador central de metal, titânio ou plástico, com diâmetros e comprimentos correspondentes aos das limas estandardizadas ISO, recobertos por guta percha de fase á que, ao ser aquecida, torna-se plastificada e aderente ao carregador, 0 qual é levado ao interior do canal radicular com um movimento único e pressão firme, em direção apical, no momento da obturação (SANTA CECÍLIA; M ORAES,; BERBERT 1995; BERTRAND et al., 1997; PERTOT, 2000; VALOIS; CASTRO, 2001).

Berger (1995) salienta que Thermafil é uma técnica indicada para canais curvos e atrésicos, onde há uma dificuldade de adaptação dos cones de guta percha até o comprimento de trabalho. Recomenda que o conjunto obturador seja levado a $0,5 \mathrm{~mm}$ do comprimento de trabalho, devido à sua facilidade de alcançar a região apical.

Z mener, Perruchino e Zacarias (1999) compararam a qualidade da obturação obtida por meio do sistema Thermafil, técnica híbrida e condensação lateral, sem a utilização de cimento endodôntico selador, através da observação dos cortes dos três terços com uma lupa estereoscópica. 0 s resultados demonstraram que as técnicas Thermafil e híbrida promoveram uma massa de guta percha mais homogênea e adequadamente distribuída em todo espaço e perímetro radicular.

Bertrand e colaboradores (1997) advertem quanto à possibilidade de ocorrência do deslocamento inadvertido da guta percha no "carrier" durante a inserção do Thermafil, o que pode contribuir para o aumento da incidência de infiltração apical. Relatam a dificuldade de detecção radiográfica desse fenômeno de escoamento e alertam quanto aos cuidados ao executar 0 Thermafil.

Valois e Castro (2001), ao avaliarem a adaptação do carreador plástico do Thermafil às paredes do canal radicular, concluíram que a distribuição da guta percha, carreador plástico ou cimento foi relativamente uniforme nos terços cervical e médio dos canais. Contudo, no terço apical de $43 \%$ das amostras, o carreador estava em contato direto com a parede do canal.

Santa Cecília, M oraes e Berbert (1995) observaram maior extrusão apical da obturação com o Thermafil, quando comparada à técnica da condensação lateral, e maior extravasamento nos canais amplos e retos que nos canais curvos e atrésicos. E quanto à capacidade seladora apical, Santa Cecília e colaboradores (1999) não detectaram diferenças estatísticas entre essas mesmas técnicas por meio da diafanização.

Tagger e colaboradores (1984) propuseram a técnica híbrida de Tagger, que consiste na associação de condensação lateral ativa no terço apical à compactação termomecânica de M cSpadden. Tagger, Santa C ecília e M oraes (1994) modificaram a técnica original, acrescentando um novo espaçamento e inserindo de novos cones acessórios após o uso do compactador, seguido de nova compactação e condensação axial vigorosa. Freitas e colaboradores (1996) comparam a compactação termomecânica de M cSpadden e a técnica híbrida de Tagger e não observaram diferença significativa entre elas. Contudo ambas apresentaram menor infiltração apical do que a técnica da Condensação Lateral.

Ferraz (1999) estudou in vitro o selamento marginal apical promovido pelas técnicas da condensação lateral, híbrida de Tagger 
e a técnica termomecânica, com o uso do ultrasom, e concluiu não haver diferenças estatísticas entre as três técnicas frente à infiltração marginal apical do corante. Q uanto ao aumento da temperatura na superfície radicular externa provocado por essa técnica, C unha e colaboradores (2004) observaram que é segura, pois a elevação média foi inferior a $100 \mathrm{C}$.

Este trabalho tem por objetivo avaliar comparativamente, "in vitro", o selamento apical em dentes obturados pelas técnicas híbridas de Tagger, Termafil e condensação lateral, através da infiltração linear do corante azul de metileno a 1\% em lupa estereoscópica com 8X de aumento.

\section{MATERIAIS E MÉTODOS}

Foram utilizados, neste estudo, 40 incisivos centrais superiores humanos, com rizogênese completa, obtidos no estoque da Disciplina de Endodontia da Faculdade de $O$ dontologia da UFBA. O s dentes foram limpos através de escovação e lavagem em água corrente, e mantidos em solução de timol a 0,1\%. Para uma devida hidratação, foram mantidos em estufa a $37^{\circ} \mathrm{C}$ durante 07 dias. Em seguida foram medidos com uma régua milimetrada, marcando-se com $19 \mathrm{~mm}$ de comprimento o local onde as coroas foram seccionadas. Iniciou-se o acesso endodôntico com ponta diamantada esférica KG Sorensen número 1015 em alta rotação e brocas Batt $n .014$ para remoção do cotovelo de dentina na entrada do canal. Um instrumento $\mathrm{K} \# 15$ foi introduzido em toda extensão do canal, até que sua ponta fosse visualizada no forame apical e, subtraindo-se $1 \mathrm{~mm}$, obteve-se o comprimento de trabalho. O preparo químico-mecânico foi realizado pela técnica de 0 regon, e a matriz apical estabelecida com limas \#55 ou \#60. A potência foi mantida com lima $K \# 10$, trabalhando-se $1 \mathrm{~mm}$ além do comprimento de trabalho. As substâncias químicas auxiliares utilizadas foram hipoclorito de sódio a $1 \%$ (Inodon), coadjuvado com o Endo PTC (Polidental). Após a instrumentação, os condutos foram irrigados com solução de ED TA a 17\% (Inodon), para a remoção da camada residual, seguindo-se a irrigação final com hipoclorito de sódio e tergensol. As amostras foram secas com pontas de papel absorvente e distribuídas em 5 grupos, sendo 3 grupos experimentais, com 10 amostras cada, e 2 grupos controle, cada um com 5 amostras. As amostras do Grupo I foram obturadas pela técnica da condensação lateral, utilizando-se cone principal do mesmo calibre do último instrumento utilizado no comprimento de trabalho (\#55 ou \#60) e cones secundários FF (D entsply). As amostras do G rupo II foram obturadas pela técnica híbrida de Tagger, utilizando-se cones principais ajustados no comprimento de trabalho e cones secundários FF (D entsply). Para cada dente, após a condensação lateral, foi selecionado um compactador de guta percha de tamanho adequado ( $M$ aillefer) (\#55 ou \#60), montado num contra ângulo, e um micromotor acionado no sentido horário, com velocidade rotacional de 8000 a 10000 rpm, introduzido no canal a $4 \mathrm{~mm}$ aquém, como margem de segurança. D eacordo com as variações anatômicas, foram realizadas de 2 a 4 compactações para se obter o preenchimento total dos condutos. Após a remoção dos excessos com um instrumento aquecido, realizou-se uma condensação vertical.

As amostras do G rupo III foram obturadas com o sistema Thermafil, utilizando-se os obturadores previamente selecionados pelos verificadores. Os obturadores, recobertos por guta percha alfa, foram aquecidos no forno Thermaprep Plus e introduzidos com pressão firme e constante nos condutos radiculares até o comprimento de trabalho. A haste central e os excessos de guta percha foram removidos com um calcador de Paiva aquecido.

As amostras do Grupo IV e V, controle positivo e negativo, foram instrumentadas e não obturadas. Todos os espécimes dos grupos supracitados foram selados com coltosol e impermeabilizados com adesivo epóxi (Scotch M ix $3 \mathrm{M}$ ) e esmalte para unhas, exceto $2 \mathrm{~mm}$ ao re dor do forame apical, com exceção das amostras do Grupo V, que foram impermeabilizadas em toda a sua extensão. 
Para todos os grupos experimentais, além da guta percha, foi utilizado o cimento obturador Endofill (D entsply).

Em seguida, todas as amostras foram imersas em azul de metileno a $1 \%$ e mantidas em estufa a $37^{\circ}$ por 7 dias. Posteriormente, foram lavadas em água corrente por 24 horas e removidas as impermeabilizações manualmente, com o auxílio de uma espátula lecron. Procedeu-se à confecção de sulcos longitudinais com disco de aço em baixa rotação e à clivagem com o auxílio de cinzéis. A infiltração linear do corante azul de metileno foi medida em lupa esteroscópica com 8X de aumento, com 0 auxílio de uma régua milimetrada. O s resultados obtidos foram registrados por 2 examinadores

Tabela 1 - Grupo I (Técnica da Condensação Lateral)

\begin{tabular}{cccc}
\hline Dente & $\begin{array}{c}\text { Condensação } \\
\text { lateral }\end{array}$ & $\begin{array}{c}\text { Híbrida de } \\
\text { Tagger }\end{array}$ & Thermafil \\
\hline 1 & 1,0 & 2,0 & 0,0 \\
2 & 1,5 & 1,0 & 4,5 \\
3 & 1,5 & 6,0 & 1,0 \\
4 & 1,0 & 2,0 & 2,0 \\
5 & 1,0 & 2,0 & 4,0 \\
6 & 0,0 & 1,5 & 2,5 \\
7 & 1,0 & 5,0 & 2,0 \\
8 & 4,0 & 0,5 & 0,0 \\
9 & 1,5 & 4,0 & 1,0 \\
10 & 3,5 & 0,5 & 0,0 \\
\hline
\end{tabular}

devidamente calibrados, expressando-se a quantidade de infiltração em milímetros.

\section{RESULTAD OS}

O s valores de infiltração do corante azul de metileno (em $\mathrm{mm}$ ), anotados pelos examinadores, são demonstrados na tabela a seguir:

Empregou-se a correlação de Spearman para verificar 0 grau de concordância entre os dois examinadores, demonstrando-se não haver diferença significativa entre eles. Portanto, para
Tabela 2 - Valores médios de infiltração

\begin{tabular}{lc}
\hline & $\begin{array}{c}\text { Infiltração Mediana } \\
\text { (mm) }\end{array}$ \\
\hline $\begin{array}{l}\text { Grupo I } \\
\text { (T. Cond. Lat.) }\end{array}$ & 1,25 \\
Grupo II & \\
(T. H. Tagger) & 2,0 \\
$\begin{array}{l}\text { Grupo III } \\
\text { (Thermafil) }\end{array}$ & 1,5 \\
\hline Nota: $p=0,494 / P>0,05$ &
\end{tabular}

análise estatística, utilizaram-se apenas os valores do examinador sênior.

Para a comparação dos valores de infiltração do azul de metileno obtidos em milímetros, utilizou-se a prova de Kruskal - Wallis. As médias encontradas estão na tabela 2. Como 0 valor $p=0,494$, considera-se que não houve diferença estatisticamente significante entre os 3 grupos experimentais.

\section{DISCUSSÃO}

Embora várias técnicas tenham sido propostas para a obturação do sistema de canais radiculares, a técnica da condensação lateral continua sendo a mais difundida e empregada na atualidade. Seu uso não requer equipamento especializado, é de baixo custo e de simples execução. Entretanto, muitos autores recomendam as técnicas termoplastificadas, justificando seu uso por considerarem haver melhor a adaptação da guta percha à parede dentinária, maior homogeneidade da massa obturadora, e bom selamento apical (FREITAS et. al., 1996; SANTA CECÍLIA et al., 1999; LEVITAN; H IMEL; LUCKEY, 2003; CUNHA et. al., 2004).

Um dos requisitos mais solicitados pelos profissionais durante a obturação do canal radicular é o menor consumo de tempo e de material. 0 sistema Thermafil preenche esses requisitos e surge como uma alternativa rápida e simples de obturação (BERGER, 1995; PERT OT, 2000). Contudo, é possível a ocorrência de sobreobturações e deslocamento inadvertido da guta percha durante o procedimento obturador, ficando apenas o carreador plásti- 
co em contato com as paredes do canal radicular (BERTRAND et al., 1997; VALOIS; CASTRO, 2001; JARRETT et al., 2004). A pesar de não ser objetivo do estudo, observou-se que, no grupo II, $20 \%$ das amostras apresentaram sobreobturações, concordando com a ressalva desses autores. Vale salientar que, neste estudo, foram avaliados incisivos centrais superiores, cujos canais amplos e retos têm maior suscetibilidade à extrusão apical, conforme é mencionado também por Santa C ecília, M oraes e Berbert.(1995).

$0 \mathrm{~s}$ resultados obtidos neste trabalho de monstraram, mais uma vez, que as técnicas obturadoras apresentaram algum grau de infiltração, porém, sem diferenças estatísticas significantes $(p>0,05)$ (FERRAZ, 1999; SAN TA CECÍLIA et al., 1999; LEONARDO et al, 2004). Entretanto, Freitas e colaboradores (1996) observaram que, na condensação lateral, houve maior infiltração de corante em relação à técnica híbrida de Tagger.
$\mathrm{N}$ ão obstante, no grupo obturado pela técnica da condensação lateral ( $G$ rupo I), foi encontrada a menor média de infiltração $(1,25 \mathrm{~mm})$, apesar de não se apresentar diferença estatisticamente significante. Assim, esses resultados justificam, ainda, a grande utilização dessa técnica de obturação, mesmo após 0 surgimento de novos sistemas obturadores.

0 método empregado para avaliar a infiltração apical foi a visualização da penetração do corante azul de metileno a $1 \%$, por se tratar de um método fácil, acessível e muito utilizado (FREITAS et al., 1996; LEONARDO et al., 2004).

\section{CONCLUSÃO}

D e acordo com a metodologia empregada, pôde-se concluir que todas as três técnicas de obturação analisadas apresentaram algum grau de infiltração apical, não havendo diferença estatística significante entre elas.

\title{
Evaluation of theapical sealingin teeth filled using Lateral C ondensation, Tagger'sH ibrid and Thermafil techniques
}

\begin{abstract}
Sealing root canal system is necessary to maintain the disinfection attained by the preparation. Several root filling techniques and sealers have been presented to reach this requisite. The aim of this study was to evaluate the apical sealing in teeth filled using L ateral C ondensation, Tagger's H ibrid and Thermafil techniques analyzing quantitatively the leakage of the $M$ ethylene Blue dye. The canals of 40 extracted teeth were endodontically prepared and separated into three experimental groups according to the filling technique used, and into two control groups. There was no statistically significant difference among the three root canal filling techniques evaluated.
\end{abstract}

Keywords. Endototics methods, root canal obturation, dental leakage.

\section{REFERÊNCIAS}

BERGER, C.R. Thermafil: avaliação clínica. R.0 dontol. UNICID, São Paulo, v.7, n.1, p.19-26, jan./jun. 1995.
BERTRAND, M.F. et al. Removal of Thermafil root canal filling material. J. Endod., Baltimore, v.23, n.1, p.54-57, Jan. 1997. 
CUNHA, R.S. et al. Avaliação in vitro da alteração de temperatura na superfície radicular externa durante a obturação do sistema de canais radiculares pela $T$ écnica $H$ íbrida de Tagger e $O$ nda C ontínua de C ondensação. JBE: J. Bras. Endod., Curitiba, v.5, n.18, p.201-206, 2004.

EST RELA, C. Ciência endodôntica. São Paulo: Artes M édicas, 2004.

FERRAZ, J.A.B. Estudo "in vitro" da capacidade de selamento marginal apical promovido por três técnicas de obturação de canais radiculares. 1999. 102f. Dissertação (M estrado)- Faculdade de 0 dontologia de Ribeirão Preto, Universidade de São Paulo, Ribeirão Preto, 1999.

FREITAS, R.M. et al. Análise in vitro do selamento apical proporcionado pela técnica híbrida de Tagger: original e modificada. $\mathbf{R}$. Bras. 0 dontol., Rio de Janeiro, v.53, n.5, p.25, set./out. 1996.

JARRETT, I.S. et al. Percentage of canals filled in apical cross sections: an in vitro study of seven obturation techniques. Int. Endod. J., London, v.37, p.392-398, 2004.

LEO N ARD O, M .L.; LEAL, J.M. Endodontia: tratamento de canais radiculares. 3.ed. São Paulo: Panamericana, 1998.

LEONARDO, M.R. et al. Effect of different rotary instrumentation techniques and thermoplastic filling on apical sealing. J. Appl. 0 ral Sci., Bauru, v.12, n.1, Jan./M ar. 2004.

LEVITAN, M .E.; H IM EL, V.T.; LUCKEY, J.B. The effect of insertion rates on fill length and adaptation of a thermoplasticized gutta-percha technique. J. Endod., Baltimores, v.29, n.8, Aug. 2003.

M IRAN ZI, M.A.S. et al. Avaliação in vitro em canais artificiais curvos de duas técnicas de obturação termoplastificadas em relação à técnica da condensação lateral. JBE: J . Bras.Endo/Perio, Curitiba, v.1, n.1, 2000.
PERTOT, W. How to use Thermafil successfully. Endod. Pract., Shenley, M ay 2000.

SANTA CECÍLIA, M. et al. Selagem apical propiciada pela técnica Thermafil em canais retos e curvos. R.Bras.0 dontol., Rio de Janeiro, v.56, n.2, mar./abr., 1999.

SANTA CECÍlIA, M.; MORAES, I.G. de; BERBERT, A. Extrusão apical de material obturador observado na técnica Thermafil. R.Fac. Odontol. Bauru, Bauru, v.3, n.1/4, p.89-93, jan./dez. 1995.

SIQUEIRA JR, J.F.; GARCIA FILHO, P.F.; FRAGA, R.C. Influência de algumas modificações na técnica de condensação lateral no selamento apical. R.Bras.0 dontol., Rio de Janeiro, v.50, n.4, jul./ago. 1993.

TAGGER, M. et al. Evaluation of the apical seal produced by a hibrid root canal filling method, combining lateral condensation and thermatic compaction. J. Endod., Baltimore, v.10, n.7, p.299-302, July 1984.

TAGGER, M ;; SANTA CECílIA, M .; M ORAES, I.G. Técnica híbrida de Tagger: modificações do método original. RGO, Porto Alegre, v.42, n.1, p.207-208, jul./ago. 1994.

VALE, M.S. do; NEUVALD, L.R.; BRAM ANTE, C.M. Avaliação da qualidade de obturação de três técnicas da guta-percha empregadas em dentes com reabsorção dentinária interna. JBE: J. Bras. Endo/Perio, Curitiba, v.2, n.6, p.240-245, jul./set. 2001.

VALOIS, C.R.A.; CASTRO,A.J.R. An in vitro study of the adaptation of Thermafil plastic carrier to canal walls. Braz. Endod. J., C uritiba, v.5, n.1/2, p.32-36, 2001.

ZMENER， 0 .; PERRUCHINO， R.; ZACARIAS, M. Análisis de la calidad de la obturación endodóntica obtenida por dos técnicas de gutapercha termoplastificada. R. Fac. O dontol., Asunción, v.13, n.10, p.49-54, oct. 1999. 